\title{
Programa de prevenção de internação para idosos na saúde suplementar: um relato de caso
}

\author{
Health Program for the prevention of hospitalization of the elderly in the private \\ health care sector: a case study

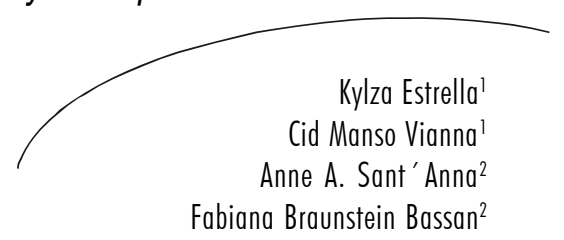

Resumo

Introdução: Este trabalho visa a apresentar um programa de prevenção de internação hospitalar para idosos na saúde suplementar e descrever as características da população sob intervenção do mesmo. Método: o programa realiza a captação de indivíduos de risco, com idade maior ou igual a 65 anos, aplicando um questionário de risco para internação hospitalar. Indivíduos de risco são contatados por telefone e convidados a participar do programa. Idosos que recusam o programa permanecem em cuidado usual, e os que o aceitam são visitados por enfermeira, em ambiente domiciliar para uma avaliação multidimensional. De acordo com essa avaliação, idosos são distribuídos em grupos funcionais que vão determinar seu acompanhamento por equipe multidisciplinar. Resultados: 627 indivíduos aceitam o programa e 392 recusam. O sexo feminino é prevalente em ambos os grupos, assim como a idade média é semelhante. A maioria é casada, $19 \%$ das mulheres vivem sós, em oposição a $9 \%$ dos homens. Percentual de analfabetos é menor do que outros estudos; $67 \%$ dos usuários do programa apresentam têm três ou mais doenças crônicas; 49,6\% apresentam limitação funcional em ao menos uma atividade de vida diária, enquanto $20 \%$ apresentam dependência total. Conclusão: É marcante a dependência funcional nos idosos do programa, o que fala a favor da presença

\author{
Palavras-chave: \\ Idoso. Prevenção \& \\ Controle. Assistên- \\ cia Domiciliar. \\ Saúde Suplementar. \\ Planejamento de \\ Assistência ao \\ Paciente. Planeja- \\ mento Assistencial
}

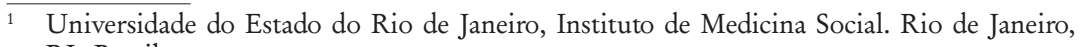
RJ, Brasil

2 Grupo Amil. Duque de Caxias, RJ, Brasil

Correspondência / Correspondence

Kylza Estrella

Universidade do Estado do Rio de Janeiro, Instituto de Medicina Social

Rua São Francisco Xavier, 524, $7^{\circ}$ andar, bloco D - Maracanã

20559-900 - Rio de Janeiro - RJ

E-mail: kylza.estrella@terra.com.br 
de seleção adversa na adesão ao programa. A diferença para escolaridade sugere uma representação da classe socioeconômica na saúde suplementar. Sugerese que modelos assistenciais de atenção integral à saúde do idoso sejam desenvolvidos na saúde suplementar.

\section{Abstract}

Introduction: This paper aims to present a program on prevention of hospitalization for the elderly in the health insurance and describe the characteristics of the population under the same intervention. Method: the program applies a screening questionnaire for those of 65 years old and above aiming to identify individuals at risk for hospitalization. Those at risk are contacted by phone and invited to participate in the program. Elderly who refuse the program are kept under usual care. Individuals who accepted the program are visited by a nurse, at their houses, for a multidimensional health evaluation. According to the latter, individuals are distributed in functional groups which will determine their health care model by the program. Results: 627 accepted the program and 392 refused it. Female is prevalent in both groups as the average age is similar for both. The majority is married, $19 \%$ of women live alone as opposed to $9 \%$ of men. The percentage of analphabets is smaller than other studies; $67 \%$ of program users have 3 or more chronic diseases; $49.6 \%$ have limited functional activity for at least one activity of daily living and $29 \%$ have total dependence. Conclusion: There is an important functional dependence in the elderly under the program, suggesting that there is an advertion selection criteria for those who accept the program. The literacy difference may be justified by the social economical class of the elderly in the private health care sector. It is suggested that integrated health care models for the elderly should be developed in the private care sector.
Key words: Aged.

Prevention \& control.

Home Nursing.

Supplemental Health.

Patient Care Planning.

Prevention. Home

Care. Private Health

Sector. Health

Planning.

\section{INTRODUÇÃO}

O envelhecimento populacional é uma realidade no cenário mundial. Projeções demográficas apontam que o Brasil será, até 2025, o sexto país do mundo em número de idosos, um contingente estimado em 32 milhões de pessoas. ${ }^{1}$ Todavia, ainda é grande a desinformação a respeito da saúde do idoso e das particularidades e desafios do envelhecimento populacional para os serviços de saúde em nosso contexto social. Essa faixa etária tem grande impacto no uso dos serviços de saúde, uma vez que, para qualquer indicador de utilização dos serviços de saúde, os idosos são os grandes usuários. ${ }^{2}$ No que diz respeito às internações hospitalares, os idosos possuem elevadas taxas de internação e maior tempo médio de ocupação do leito hospitalar, quando comparados a outros grupos etários. ${ }^{3}$ Ainda neste segmento etário, existe uma heterogeneidade marcante: por um lado, $80 \%$ da população idosa pode ser considerada saudável, por 
outro, de 10 a $25 \%$ são identificados como sujeitos frágeis, que necessitam de cuidados intensivos. ${ }^{4}$

Nesse contexto, a mudança de paradigma de atenção à saúde da população idosa é imprescindível. Saúde na terceira idade não está relacionada somente à ausência de doença, mas sim à capacidade funcional. ${ }^{2} \mathrm{~A}$ perda da capacidade funcional está associada à maior hospitalização e ao aumento da mortalidade. ${ }^{5-7} \mathrm{O}$ modelo de atenção à saúde destinado ao idoso deve ser capaz de identificar indivíduos fragilizados, isto é, aqueles com maior risco de desenvolver incapacidade funcional, assim como acompanhar de forma eficiente os de menor risco, paralelamente ao desenvolvimento de ações e atividades de educação e promoção da saúde. ${ }^{3}$

Este cenário é reproduzido no ambiente da saúde suplementar, sendo agravado por um modelo assistencial reconhecidamente hospitalocêntrico e centrado na doença ${ }^{8} \mathrm{e}$ tendo, na atualidade, um complexo de atividades em ação desconexa, geradoras de alto custo nos serviços de saúde. $\mathrm{Na}$ saúde suplementar, o percentual de cobertura da população idosa é importante, sendo que a faixa etária acima de 70 anos representa a cobertura mais elevada na população brasileira. ${ }^{9}$ Justificado por esta realidade, foi desenvolvido em uma operadora de saúde, a Dix Saúde no Rio de Janeiro, um programa específico para os idosos. O objetivo deste trabalho é apresentar o programa e realizar uma descrição das características da população sob intervenção do mesmo.

\section{O PROGRAMA}

O programa se inicia na captação de indivíduos (idosos com idade maior ou igual a 65 anos) e identificação do grupo de risco, utilizando um questionário de risco para internação hospitalar. ${ }^{10} \mathrm{O}$ questionário é aplicado através de contato telefônico, dirigido ao idoso, cuidador ou responsável pelo mesmo. Esse questionário resulta em um coeficiente (com valores de 0,07 a 0,78); quando valores acima de 0,5 identificam indivíduos com maior probabilidade para internação hospitalar. ${ }^{10}$ Desta maneira, em ordem decrescente de acordo com os resultados deste coeficiente, indivíduos de maior risco são priorizados para o cuidado imediato.

Indivíduos com este perfil de risco são contatados por telefone pelo programa, que é apresentado como uma proposta de um modelo de assistência ao idoso com equipe multidisciplinar, com foco em prevenção. Nesta fase, também é esclarecido que o programa é um benefício, não agregando custo algum para o indivíduo nem tampouco limitação na utilização dos serviços disponibilizados pela operadora de saúde. Nesta etapa, os indivíduos de risco dividem-se em usuários que recusam o programa e permanecem no cuidado usual e os que aderem e iniciam o acompanhamento pelo programa.

Para o grupo com adesão, é marcada a primeira visita de enfermagem na residência do usuário, para a realização de uma avaliação multidimensional. Para todos os 
pacientes com dependência parcial e autonomia, uma nova avaliação multidimensional é programada a cada 12 meses.

Esta avaliação é um protocolo fechado, que levanta a história psicossocial e clínicofuncional do idoso, com uso de instrumentos consolidados nas áreas de Geriatria e Gerontologia, validados e amplamente utilizados no Brasil. ${ }^{11}$ Escalas são utilizadas neste momento, sendo elas: 1) Katz ${ }^{12}$ para atividades básicas de vida diária; 2) Lawton ${ }^{13}$ para as atividades instrumentais de vida diária; 3) Mini-exame do estado menta $1{ }^{14}$ para avaliação cognitiva; e 4) Escala de Depressão Geriátrica para mapeamento de depressão. ${ }^{15}$ De acordo com o quadro de cada paciente, a conduta de enfermagem nessa primeira visita envolve orientação ao paciente e sua família, discutindo questões relacionadas ao envelhecimento saudável, preservação da capacidade funcional, participação social e orientação quanto a rede assistencial proposta pelo programa.

Esse protocolo é posteriormente avaliado pela enfermeira(o), em conjunto com um(a) médico(a) geriatra da equipe. A partir dessa análise, que descreve os diagnósticos psicossocial e clínico-funcional, é definida uma conduta de acompanhamento pelo programa. Esse acompanhamento está embasado no entendimento de que a dependência funcional é preditora de mortalidade e de utilização de serviços de saúde. ${ }^{5,6}$ Ramos $^{5}$ demonstrou que a dependência severa aumenta em três vezes o risco de mortalidade e que resultados de $\mathrm{MEEM}^{14}$ menores ou iguais a 18 têm quase o dobro de risco para mortalidade daqueles com valores superiores.

A partir da avaliação das escalas de Katz ${ }^{12}$ e MEEM, ${ }^{14}$ é realizada uma estratificação dos idosos em grupos, que irá definir a forma de acompanhamento pelo programa. Os pacientes com dependência total (dependência em 2 ou mais atividade de vida diária na escala de Katz ${ }^{12}$ ou MEEM ${ }^{14}$ menor ou igual a 18) recebem visita de enfermagem mensalmente, sendo denominados grupo $1 \mathrm{e} 1^{\mathrm{a}}$ (este também com visita médica domiciliar). Indivíduos com dependência parcial (dependência em 1 atividade básica de vida diária ou $\mathrm{MEEM}^{14}$ entre 19 e 23) recebem visita de enfermagem bimensal, intercalada com acompanhamento telefônico realizado por enfermeira, denominados grupo 2. E pacientes com autonomia (sem dependência nas atividades de vida diária e MEEM $^{14}$ maior que 23) recebem monitoramento telefônico por enfermeira trimestralmente, sendo denominados grupo 3 . Os pacientes neste último grupo, mas com idade igual ou maior que 80 anos, possuem visita de enfermagem trimestral intercaladas com monitoramento telefônico e são denominados grupo $2 \mathrm{~B}$.

As visitas e contatos telefônicos da enfermagem obedecem a um protocolo com dados relevantes ao acompanhamento geriátrico/gerontológico, que reitera as medidas preconizadas na visita de enfermagem inicial. Os pacientes restritos, que não possuem condições clínicas ou suporte social adequado para que sejam levados a um consultório médico, recebem visitas médi- 
cas programadas no domicílio. No caso de intercorrências, visitas de enfermagem e/ ou médicas podem ser antecipadas, respeitando-se a individualidade e demanda clínica do caso.

Os demais profissionais da equipe (fisioterapeuta, nutricionista, assistente social e psicóloga) atuam eletivamente de acordo com avaliação da necessidade identificada pela enfermeira. Os encaminhamentos para esses profissionais obedecem a critérios preestabelecidos. A fisioterapia atua em três processos distintos: reabilitação (para pacientes que, após a internação hospitalar, apresentam declínio funcional, sequela de acidente vascular cerebral, etc.); prevenção de quedas (pacientes com relato de 2 ou mais quedas por semestre), alterações na marcha e ainda para treino de cuidador, quando o cuidador do paciente idoso é orientado para realização de exercícios passivos, cuidados na transferência do paciente, etc.

A nutrição visita pacientes desnutridos, obesos, diabéticos, com úlceras de decúbito, em uso de gastrostomia, etc. Acompanhamento psicológico é indicado para pacientes com depressão, dificuldades na relação familiar, etc. A assistente social acompanha famílias e pacientes na tentativa de buscar as condições necessárias para a assistência e qualidade de vida do mesmo. Todas as avaliações são devidamente registradas em prontuário eletrônico único.

No caso de internação hospitalar, o Programa mantém contato com o médico responsável pela internação, para acompanha- mento do paciente. Após a alta, é agendada uma visita de enfermagem em ambiente domiciliar e, para pacientes com visita médica, esta é antecipada.

A rotina da enfermagem na residência do usuário realiza uma integração permanente da assistência. Sintomas clínicos relevantes são comunicados à equipe médica; as orientações nutricionais realizadas pela nutricionista são reforçadas e ainda se na rotina do cuidado existe a necessidade de intervenção da assistência social ou psicologia.

\section{RESULTADOS E DISCUSSÃO}

Esta análise descritiva diz respeito aos resultados encontrados para o grupo sob intervenção do programa no momento da avaliação multidimensional para um total de 627 pacientes. O grupo que recusa a participação no programa e permanece em cuidado usual totaliza 392 pacientes.

\section{CARACTERÍSTICAS SOCIODEMOGRÁFICAS}

A presença do sexo feminino é prevalente em ambos, com $79 \%$ no grupo sob intervenção e $77 \%$ de mulheres no grupo que recusa o programa. A idade média é semelhante em ambos os grupos, com 76,6 anos para o grupo sob intervenção e 76,1 anos para o grupo em cuidado usual. Idosos jovens ( 65 a 70 anos) têm um percentual maior no grupo em cuidado usual e o grupo mais idoso, acima de 80 anos, prevalece no grupo sob intervenção. É fato que, no ambien- 
te da saúde suplementar, ocorre uma prevalência do sexo feminino, ${ }^{8}$ assim como as mulheres são reconhecidamente maiores usuárias dos serviços de saúde de um modo geral, ${ }^{11}$ sendo este padrão espelhado na operadora. A partir da análise das características de sexo e idade, as demais variáveis irão contemplar apenas o grupo sob intervenção do programa.

Para fins desta pesquisa, foi definido como casado o indivíduo em união formalizada ou não, isto é, casais idosos que vivem juntos. A maioria dos idosos do programa vive acompanhada e é casada, mantendo uma característica dos idosos no Brasil. ${ }^{16,17}$ Idosos casados compõem 51\% do grupo, e há um importante percentual de viúvos (37\%). Indivíduos separados representam um grupo menor (8\%), seguidos pelos solteiros (4\%). Aqui o gênero faz diferença, uma vez que entre as mulheres do programa, 19\% vivem sós, em contraste com os homens, com apenas $9 \%$ vivendo sozinhos. Este aspecto acompanha o quadro descrito no Brasil, uma vez que tem sido demonstrado que as mulheres idosas vivem sós e os homens casam-se com mulheres mais jovens. ${ }^{16,18}$

No que concerne às condições de moradia, isto é, se vivem sós, acompanhados ou com um cuidador, os idosos vivem em sua maioria acompanhados (78\%); $17 \%$ vivem sós e apenas $5 \%$ vivem na companhia de um cuidador remunerado pela família. Entre os que vivem sozinhos, 29\% têm idade maior ou igual a 80 anos, novamente acompanhando um predicado dos idosos. Lebrão, ${ }^{19}$ em levantamento no município de São Paulo, outra grande capital brasileira, demonstrou que $21,5 \%$ dos idosos octagenários vivem sós.

A população do programa reside prioritariamente no município do Rio de Janeiro $(73 \%)$, nos bairros da Tijuca $(8,2 \%)$ e Copacabana (7,9\%). O município de Niterói ocupa o segundo lugar, onde residem $14,3 \%$ indivíduos do grupo. A Baixada Fluminense tem o menor percentual de idosos, com $13 \%$ dos usuários, em sua maioria residindo em Duque de Caxias (8,5\%).

A renda foi levantada levando-se em conta a existência de trabalho remunerado ou aposentadoria para os idosos, considerando a renda do usuário idoso e não da família. A renda média mensal encontrada foi de $\mathrm{R} \$ 674,74$ (desvio padrão=1.104,26) e $25 \%$ do grupo relatam não ter renda; $50 \%$ dos usuários têm renda abaixo da média; $25 \%$ têm renda maior ou igual a $\mathrm{R} \$ 800,00$, sendo o valor máximo de renda de $\mathrm{R} \$$ $10.000,00$.

Escolaridade foi avaliada em anos de estudo, divididos em quatro grupos: analfabeto, de $\mathrm{O}$ a 4 anos de estudo, de 5 a 8 anos e mais que 8 anos de estudo. Na relação com gênero, na condição de analfabeto há um percentual maior para o sexo feminino $(3,8 \%)$ e menor $(2,3 \%)$ para o masculino, diferentemente de outros estudos, onde este percentual é maior. ${ }^{19}$ Este achado aponta para um reflexo das classes sociais a que os idosos pertencem, de um grupo inserido na saúde suplementar. Este percentual fica mais próximo ao encontrado em outras capitais latinas: 
Buenos Aires, com 3,3\% de idosos analfabetos e Montevidéu, com 4,2\%. ${ }^{19}$ Em relação a gênero e escolaridade, o encontrado no programa comunga com demais estudos, ${ }^{16}$ segundo os quais a população feminina tem sempre menor escolaridade: $17,4 \%$ das mulheres têm mais do que oito anos de estudo em relação a $30 \%$ dos homens.

\section{AVALIAÇÃO CLÍNICO-FUNCIONAL}

A descrição da morbidade do grupo sob intervenção é apresentada seguida por uma análise das escalas de avaliação relativas às atividades de vida diária, depressão e cognição. Serão apresentados os grupos funcionais que representam a estratificação dos idosos segundo sua autonomia ou dependência e modelo de acompanhamento pelo programa.
A comorbidade é uma característica da população idosa, sendo também um atributo dos usuários do programa. O quadro de morbidade apresentado é aquele da população idosa em geral, com alta prevalência de doenças crônicas, com destaque para a hipertensão arterial e doenças articulares. ${ }^{17,19} \mathrm{~A}$ avaliação dos diagnósticos apresenta $67 \%$ dos usuários com três ou mais doenças crônicas, e apenas $11 \%$ apresentam apenas um diagnóstico. A tabela 1 mostra a distribuição das doenças crônicas e sistemas mais acometidos, segundo sexo. Para fins desta pesquisa, definiram-se como doenças articulares, o grupamento das doenças artrite, artrose e reumáticas. A doença de maior prevalência é a hipertensão, seguida por doenças articulares nas mulheres e as cardiopatias nos homens.

Tabela 1 - Frequência (\%) de doença crônica segundo sexo. Rio de Janeiro, RJ, 2008.

\begin{tabular}{lcc}
\hline Doença crônica & Feminino $\mathrm{N}=491$ & Masculino $\mathrm{N}=131$ \\
Hipertensão arterial & 54,7 & 49,1 \\
Doenças articulares & 30,8 & 17,6 \\
Cardiopatias & 18,3 & 23,7 \\
Osteoporose & 15,8 & 3,4 \\
Doenças digestivas & 13,4 & 9,8 \\
Diabetes & 16,7 & 13,2 \\
Sistema circulatório & 12,8 & 8,3 \\
Depressão & 10,3 & 3,1 \\
Dç. crônica pulmonar & 8,3 & 13,8 \\
Demência & 4,3 & 1,2 \\
Sequela AVC & 6,8 & 8,2 \\
Outros & 6,3 & 6,9 \\
\hline
\end{tabular}

Nota: Estão faltando dados em 5 observações nesta variável. 


\section{AVALIAÇÃO DAS ATIVIDADES DE VIDA DIÁRIA}

$\mathrm{Na}$ análise de resultados para as atividades básicas de vida diária (escala Katz), observa-se uma divisão em dois grupos: um primeiro, em que metade dos idosos
$(49,6 \%)$ apresenta limitação funcional em ao menos uma atividade de vida diária (Katz igual ou abaixo de B); e um segundo grupo $(50,4 \%)$, com autonomia, sem limitações funcionais que impeçam seu autocuidado, (Katz A), conforme apresentado na tabela $2 .^{12}$

Tabela 2 - Distribuição da frequência(\%) dos resultados para a escala Katz. Rio de Janeiro, RJ, 2008.

\begin{tabular}{lccc}
\hline Katz & Frequência (n) & Percentual (\%) & Cumulativo (\%) \\
A & 313 & 50,4 & 50,4 \\
B & 181 & 29,15 & 78,55 \\
C & 27 & 4,35 & 84,9 \\
D & 9 & 1,45 & 85,35 \\
E & 9 & 1,45 & 86,80 \\
F & 35 & 5,64 & 92,43 \\
G & 47 & 7,57 & 100,00 \\
Total & 621 & & 100,00 \\
\hline
\end{tabular}

Nota: Estão faltando dados em 6 observações nesta variável

$\mathrm{A}=$ independência em atividade de alimentação, em continência, em transferência, em higiene pessoal, em vestuário e em tomar banho.

$\mathrm{B}=$ Independência em todas as atividades de vida diária, exceto uma.

$\mathrm{C}=$ Independência em todas as atividades de vida diária, exceto tomar banho e uma outra função.

$\mathrm{D}$ = Independência em todas as atividades diárias, exceto tomar banho, vestir-se, "ir ao banheiro" e uma outra função.

$\mathrm{E}=$ Independência em todas as atividades diárias, exceto tomar banho, vestir-se "ir ao banheiro, transferir-se e uma outra função.

$\mathrm{F}=$ Independência em todas as atividades diárias, exceto tomar banho, vestir-ser, "ir ao banheiro", transferir-se e uma outra função

$\mathrm{G}=$ Dependência em todas as seis atividades.

Outros = dependente em, pelo menos, duas funções, porém não classificável como C, D, E, ou F. 
Analisando-se esta dependência entre os idosos que referiram dificuldades, verifica-se que indivíduos com limitação em somente uma atividade (Katz B) perfazem 29\% do grupo, sendo identificados no programa como idosos com dependência parcial. Quando é avaliado um nível mais complexo de dependência, com limitações em duas ou mais atividades (valores de Katz com resultado C e abaixo), em que a dependência define a presença de um familiar ou cuidador na rotina do indivíduo (uma vez que, a partir desta classificação, há necessidade de assistência no banho diário), observa-se que $20 \%$ dos idosos com limitações estão aí estratificados, correspondendo ao grupo de dependência total na classificação do programa. ${ }^{12}$

Relacionando-se a avaliação de dependência com faixa etária nos cortes utilizados para o programa (tabela 3 ), verifica-se que $69 \%$ do grupo com dependência total (Katz C-G) tem idade maior ou igual a 80 anos, discriminando uma relação esperada entre idade e dependência. Entre os resultados de Katz para sexo, na tabela 4 destacam-se a autonomia (Katz A) no grupo masculino, com $68 \%$ de homens idosos independentes para as atividades de vida diária, versus $46 \%$ das mulheres.

Tabela 3 - Distribuição (\%) dos resultados da escala de Katz e faixa etária. Rio de Janeiro, RJ, 2008.

\begin{tabular}{lllll}
\hline Katz & 65 a 70 anos & 71 a 79 anos & $\geq 80$ anos & Total \\
A (\%) & $22 \%$ & $56 \%$ & $22 \%$ & 313 \\
B (\%) & $12 \%$ & $54 \%$ & $34 \%$ & 181 \\
C-G $(\%)$ & $4.5 \%$ & $26 \%$ & $69 \%$ & 127 \\
\hline
\end{tabular}

Nota: Estão faltando dados em seis observações nesta variável.

Tabela 4 - Resultado da escala de Katz e sexo. Rio de Janeiro, RJ, 2008.

\begin{tabular}{lll}
\hline Katz & Feminino N=491 & Masculino N=130 \\
$\mathrm{A}$ & $46 \%$ & $68 \%$ \\
$\mathrm{~B}$ & $33 \%$ & $14 \%$ \\
$\mathrm{C}-\mathrm{G}$ & $21 \%$ & $18 \%$ \\
\hline
\end{tabular}

Nota: Estão faltando dados em seis observações nesta variável. 
A escala de Lawton, ${ }^{13}$ para as atividades instrumentais de vida diária, tem sete aspectos das atividades mais complexas sendo avaliados, cada um recebendo a pontuação de 1 a 3 . Valores finais para a escala vão de 7 a 21, com scores abaixo de 21 já referindo necessidade de ajuda em alguma atividade instrumental de vida diária.
Nos resultados encontrados na leitura da tabela 5 , apenas $18,8 \%$ dos idosos apresentam autonomia para a realização completa destas atividades (ou seja, com Lawton $=21$ ), ficando então $81 \%$ com necessidade de apoio ou dependência para a realização das atividades de maior complexidade na vida diária.

Tabela 5 - Distribuição da frequência (\%) dos resultados para a escala de Lawton. Rio de Janeiro, RJ, 2008.

\begin{tabular}{lccc}
\hline Lawton & Frequência (n) & Percentual \% & Cumulativo (\%) \\
7 & 31 & 4,94 & 4,94 \\
8 & 26 & 4,15 & 9,09 \\
9 & 26 & 4,15 & 13,24 \\
10 & 12 & 1,91 & 15,5 \\
11 & 22 & 3,51 & 18,66 \\
12 & 10 & 1,59 & 20,26 \\
13 & 21 & 3,35 & 23,60 \\
14 & 14 & 2,23 & 25,84 \\
15 & 28 & 4,47 & 30,30 \\
16 & 24 & 3,83 & 34,13 \\
17 & 36 & 5,74 & 39,87 \\
18 & 37 & 5,90 & 45,77 \\
19 & 60 & 9,57 & 55,34 \\
20 & 162 & 25,84 & 81,18 \\
21 & 118 & 18,82 & 100,00 \\
Total & 627 & & 100,00 \\
\hline
\end{tabular}

Este é um diferencial na análise dos idosos do programa em relação aos demais estudos. A população idosa, de modo geral, tem um percentual de dependência em torno de $20 \%$ para as atividades básicas de vida diária, sendo um pouco mais elevado para as atividades instrumentais de vida diária, uma vez que têm maior complexidade..$^{19,20}$ Ao que parece, para idosos do programa há uma relação inversa, com prevalência da dependência, seja na esfera das atividades básicas para o auto cuidado, ou nas atividades instrumentais e mais complexas de vida diária. 
AVALIAÇÃO COGNITIVA E RISCO

O ponto de corte menor ou igual a 18 no $\mathrm{MEEM}^{14}$ está relacionado a fator preditor de mortalidade para idosos. ${ }^{5}$ Neste segmento, indivíduos com resultado para MEEM ${ }^{14}$ menor ou igual a 18 , no programa, são considerados com de- pendência total. A partir deste, um resultado intermediário foi definido para a dependência parcial, com escores maiores que 18 e menores que 24 , e valores ótimos para o MEEM,${ }^{14}$ maior ou igual a 24, para idosos com autonomia. Resultados da avaliação cognitiva estão descritos a seguir, na tabela 6 .

Tabela 6 - Distribuição da frequência (\%) dos resultados para o Mini Exame do Estado Mental (MEEM). Rio de Janeiro, RJ, 2008.

\begin{tabular}{|c|c|c|c|}
\hline MEEM & FREQUENNCIA & PERCENTUAL & CUMULATIVO \\
\hline 1 & 1 & 0,16 & 0,16 \\
\hline 3 & 6 & 0,96 & 1,12 \\
\hline 6 & 2 & 0,32 & 1,44 \\
\hline 7 & 2 & 0,32 & 1,76 \\
\hline 8 & 1 & 0,16 & 1,92 \\
\hline 9 & 7 & 1,12 & 3,04 \\
\hline 10 & 4 & 0,64 & 3,68 \\
\hline 11 & 2 & 0,32 & 4,00 \\
\hline 12 & 4 & 0,64 & 4,64 \\
\hline 13 & 9 & 1,44 & 6,08 \\
\hline 14 & 6 & 0,96 & 7,04 \\
\hline 15 & 10 & 1,60 & 8,64 \\
\hline 16 & 9 & 1,44 & 10,08 \\
\hline 17 & 8 & 1,28 & 11,36 \\
\hline 18 & 13 & 2,08 & 13,44 \\
\hline 19 & 18 & 2,88 & 16,32 \\
\hline 20 & 16 & 2,56 & 18,88 \\
\hline 21 & 29 & 4,64 & 23,52 \\
\hline 22 & 21 & 3,36 & 26,88 \\
\hline 23 & 38 & 6,08 & 32,96 \\
\hline 24 & 49 & 7,84 & 40,80 \\
\hline 25 & 74 & 11,84 & 52,64 \\
\hline 26 & 73 & 11,68 & 64,32 \\
\hline 27 & 59 & 9,44 & 73,76 \\
\hline 28 & 64 & 10,24 & 84,00 \\
\hline 29 & 50 & 8,00 & 92,00 \\
\hline 30 & 50 & 8,00 & 100,00 \\
\hline Total & 627 & 100,00 & \\
\hline
\end{tabular}

Nota: Resultados para MEEM 2,4 e 5 não foram encontrados. 
Para o programa, segundo o critério isolado do MEEM, ${ }^{14} 13 \%$ são definidos como parte do grupo de dependência total e 20\% com dependência parcial, posteriormente com acompanhamento distintos. Desta maneira, 67\% dos idosos apresentam autonomia neste critério.

$\mathrm{Na}$ tabela 7, em relação ao sexo e resultados para MEEM, observa-se que am- bos os sexos apresentam os menores percentuais para resultados condizentes com risco (MEEM menor ou igual a 18). Assim como, para ambos os sexos, observase a maior frequência para indivíduos com resultados ótimos do MEEM (maior ou igual a 24). Na relação MEEM e faixa etária, verifica-se que $63 \%$ do grupo mais idoso têm resultado para MEEM menor ou igual a $18 .{ }^{14}$

Tabela 7 - Distribuição dos resultados para o MEEM e sexo. Rio de Janeiro, RJ, 2008.

\begin{tabular}{lcc}
\hline MEEM & Feminino $\mathrm{N}=496$ & Masculino $\mathrm{N}=131$ \\
$<=18$ & $14,1 \%$ & $10,6 \%$ \\
$>18<24$ & $20,5 \%$ & $12,2 \%$ \\
$>=24$ & $64,5 \%$ & $77 \%$ \\
\hline
\end{tabular}

Em relação a sexo e idade, a distribuição das características de dependência para idosos do programa segue o verificado em demais estudos, com as mulheres e os mais idosos com pior desempenho. ${ }^{17,20}$ Da mesma forma, na relação entre declínio cognitivo e idade (reportada pelo MEEM), verifica-se que os mais idosos apresentam maior comprometimento cognitivo. ${ }^{21}$

\section{AVALIAÇÃOO DE DEPRESSÃO}

O resultado da escala de depressão (EDG), quando maior ou igual a 5, indica que a investigação a respeito de depressão deve prosseguir. Na perspectiva de que escalas são ferramentas para exploração de as- pectos de um indivíduo, sendo úteis para o mapeamento de uma população e não instrumentos para diagnósticos, o resultado da EDG no grupo sob intervenção alerta para a presença de depressão como possível diagnóstico prevalente, $39 \%$ dos idosos do programa com resultado para EDG maior ou igual a 5 .

Essa característica é relevante para fins de acompanhamento, uma vez que a depressão está vinculada à morbidade e piora de prognóstico nesta faixa etária. O fato de a escala apresentar percentual elevado e muito maior do que o diagnóstico em si aponta para uma necessidade de maior investigação dessa patologia, geralmente subdiagnosticada entre os idosos. ${ }^{22}$ 


\section{GRUPOS FUNCIONAIS}

Esta análise termina com a composição dos grupos funcionais que recebem acompanhamento de acordo com a classificação decorrente da aplicação das escalas. Para acompanhamento pelo progra$\mathrm{ma}$, ficam definidos os grupos segundo critérios de dependência total (1 e 1A), dependência parcial (2) e autonomia (3 e
2B). O fluxograma 1 apresenta definição final dos grupos funcionais e o número de usuários em cada grupo. Para cada grupo funcional, um modelo de acompanhamento contínuo para o idoso está planejado, conforme descrito anteriormente. Este processo viabiliza o planejamento das visitas com um olhar preventivo, independente das intercorrências clínicas ou não do indivíduo.

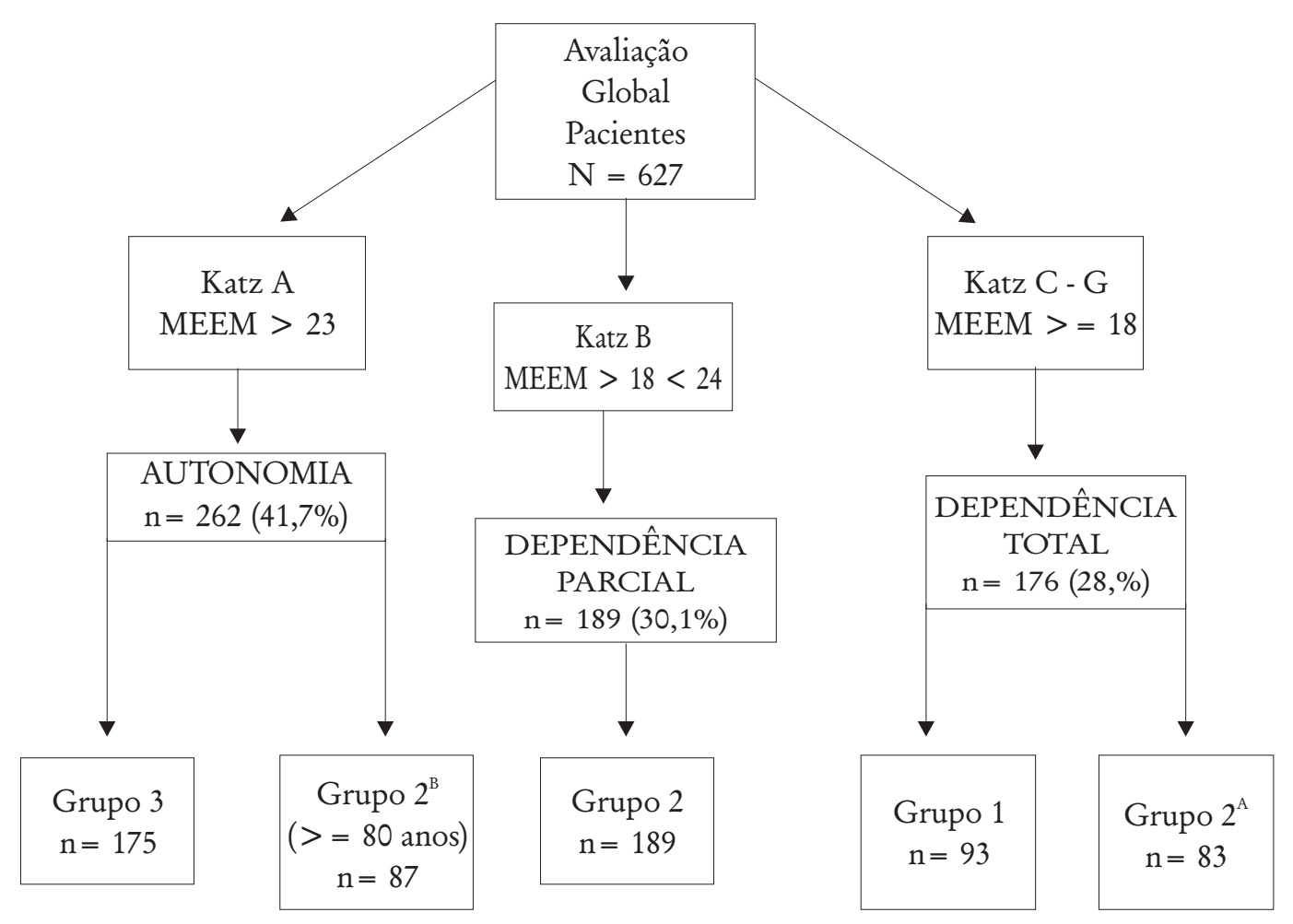

Fluxograma 1 - Classificação dos grupos funcionais e acompanhamento pelo Programa. 


\section{CONCLUSÃO}

O grupo de usuários do programa sugere características gerais semelhantes à população idosa brasileira. A exceção para tal semelhança se faz para a escolaridade, quando idosos do programa apresentam um percentual menor de analfabetos, em comparação com levantamentos em nosso meio. Este aspecto sugere uma representação da classe socioeconômica dos idosos do programa, diferente, neste sentido, de uma população geral.

Entretanto, o maior marco de diferença entre os idosos do programa e aqueles de estudos apresentados fica a cargo da avaliação de dependência funcional. Os usuários do programa apresentam maiores percentuais de dependência para atividades básicas de vida diária e para as instrumentais de vida diária, quando comparados a outras avaliações. Esse cenário caracteriza um contexto de seleção adversa, ${ }^{23}$ sugerindo que indivíduos que aceitam o programa possivelmente o fazem por apresentarem maior risco de saúde.

O modelo do programa adota para planejamento de assistência ao idoso elemen- tos específicos preconizados para avaliação e o acompanhamento desta população. $\mathrm{O}$ processo de avaliação e estratificação se inicia na porta de entrada, quando se estabelece uma prioridade para captação dos sujeitos de maior risco. Em um segundo tempo, a avaliação multidimensional dos idosos em grupos de assistência de acordo com sua capacidade funcional viabiliza a definição de critérios para acompanhamento dos grupos.

Vale comentar que um programa de cuidado integral à pessoa idosa necessita envolver acompanhamento domiciliar, sendo inclusive previsto na Política Nacional do Idoso, de 1994. ${ }^{24}$ Todavia, esta modalidade precisa estar de fato integrada aos demais processos assistenciais, de modo a ver seus resultados amplificados tanto na reabilitação e manutenção da saúde dos pacientes, quanto para atingir os melhores resultados de custo/efetividade. Sugere-se que modelos assistenciais de atenção integral à pessoa idosa merecem ser desenvolvidos no ambiente da saúde suplementar, plenamente justificados pelo franco envelhecimento populacional no Brasil, assim como pela demanda por serviços de saúde que vislumbrem a especificidade da população idosa. 


\section{REFERÊNCIAS}

1. Organização Mundial de Saúde (OMS). Envelhecimento ativo: uma política de saúde. Brasília: Organização PanAmericana de Saúde; 2005. 60p.

2. Gordilho A, et al. Desafios a serem enfrentados no terceiro milênio pelo setor saúde na atenção integral ao idoso. Rio de Janeiro: UnATI/UERJ; 2000. 92p.

3. Lourenço RA, et al. Assistência ambulatorial geriátrica: hierarquização da demanda. Rev Saude Publica 2005; 39(2): 311-8.

4. Fried LP, Waltson J. Frailty and failure to thrive. In: Hazzard WR, et al, editors. Principles of geriatric medicine \& gerontology. 5. ed. New York: MCGrawHill. p. 487-502.

5. Ramos LR, Simões EJ, Albert MS. Dependence in activities of daily living and cognitive impairment strongly predicted mortality in oldr urban residents in Brazil: a 2 year follow-up. J Am Geriatr Soc 2001; 49: 1168-75.

6. Siqueira $A B$, et al. Impacto funcional da internação hospitalar de pacientes idosos. Rev Saúde Pública 2004; 38(5): 687-94.

7. Sylvia ML, et al. Clinical features of highrisk older persons identified by predictive modeling. Disease Management 2006; 9(1): 56-62.

8. Malta DC, Jorge AO. Breve caracterização da saúde suplementar. In: Brasil. Ministério da Saúde. Agência Nacional de Saúde Suplementar. Duas faces da mesma moeda: microrregulação e modelos assistenciais na saúde suplementar. Rio de Janeiro: Ministério da Saúde; 2005b. p. 37-74.

9. Veras RP, et al. Características demográficas dos idosos vinculados ao sistema suplementar de saúde no Brasil. Rev Saúde Pública 2008; 42(3): 497-502.

10. Boult C, et al. Screening elders for risk of hospital admission. J Am Geriatr Soc 1993; 4: 811-7.

11. Veras RP. Terceira idade: gestão contemporânea em saúde. Rio de Janeiro: Relume Dumará; 2002. 190p.

12. Katz S, et al. Progressive development of the Index of ADL. Gerontologist 1970; 10: 20-30.

13. Lawton MP, Brody EM. Assessment of older people: self maintaining and instrumental activities of daily living. Gerontologist 1969; 9: 179-86.

14. Folstein MF, Folstein S, MacHugh PR. Mini-Mental State: a practical method for grading the cognitive state of patients for the clinician. J Psychiatr Res 1975; 12: 18998.

15. Yesavage JA, Brink TL. Development and validation of a geriatric screening scale: a preliminary report. J Psychiatr Res 1983; 17(1): 37-49.

16. Instituto Brasileiro de Geografia e Estatística. Perfil dos idosos responsáveis pelos domicílios no Brasil. Estudos e Pesquisas Informação Demográfica e Socioeconômica 2000; 9: 97p. [acesso em: mar 2008] Disponível em : URL: < http:// www.ibge.gov.br/população/idoso >

17. Ramos LR. Fatores determinantes do envelhecimento saudável em idosos residentes em centro urbano: Projeto Epidoso, São Paulo. Cad Saúde Pública 2003; 19(3): p. 793-8.

18. Berquó E. Considerações sobre o envelhecimento da população no Brasil. In: Bertolucci PHF, et al. O Mini-Exame do Estado Mental em uma população 
geral: impacto da escolaridade. Arq Neuropsiquiatr 1994; 52: 1-7.

19. Lebrão ML, Laurenti R. Saúde, bem-estar e envelhecimento: o estudo SABE no Município de São Paulo. Revista Brasileira de Epidemiologia 2005; 8(2): 127-41.

20. Veras RP. País jovem com cabelos brancos: a saúde do idoso no Brasil. Rio de Janeiro: Relume Dumará; 1994. 224p.

21. Cerqueira A. Deterioração cognitiva e depressão. In: Lebrão ML; Duarte Y, orgs. O Projeto Sabe no Município de São Paulo: uma abordagem inicial. Brasília:
OPAS/MS; 2003. p.143-65.

22. Blazer DG. Epidemiologia dos tanstornos psiquiátricos no idoso. In: Busse EW; Blazer DG. Psiquiatria Geriátrica. Porto Alegre: Artes Médicas; 1994. p. 167-83.

23. Cutler D; Zeckhauser R. Adverse selection in health insurance. Geneva: National Bureau of Economic Research; 1997. 29 p. (Working Paper 6107).

24. Brasil. Lei n 8.842 de 4 de Janeiro de 1994. Dispõe sobre a Política Nacional do Idoso, cria o Conselho Nacional do Idoso e dá outras providências. Pub DOU, [1994 jan 05].

Recebido:25/3/2009

Aprovado:28/8/2009 